\title{
Análisis de la aplicación del pico y placa en la ciudad de Quito
}

\section{Analysis of the application of the peak and plate in the city of Quito}

\author{
Msc. Abel Polivio Remache Coyago \\ Universidad Internacional SEK Ecuador, Ecuador \\ Ing. Santiago Fernando Celi Ortega \\ Universidad Internacional SEK Ecuador, Ecuador \\ MSc. Adolfo Juan Peña Pinargote \\ Universidad Internacional del Ecuador, Ecuador
}

Autor por Correspondencia: abelbelo@ hotmail.com; santiago.celi@uisek.edu.ec; ajpena@internacional.edu.ec

Fecha de recepción: 4 de Marzo de 2017 - Fecha de aceptación: 5 de Junio de 2017

\section{Resumen}

Pese a los esfuerzos realizados por las diferentes administraciones municipales del Distrito Metropolitano de Quito, es muy notorio que el tráfico de la Ciudad, cada vez se vuelve más caótico. Se han aplicado políticas públicas que han sido tomadas como ejemplos de otros países, sin considerar las necesidades reales de la ciudad, tal es el caso del Pico y Placa, utilizado en El Distrito Federal de México, y en Bogotá, y que en vez de solucionar el inconveniente, han hecho posible que las familias de estratos económicos medios y altos, adquieran uno o más vehículos, de la misma forma, los recursos invertidos por los municipios para el control y regulación del tráfico cada vez son más altos.

Palabras claves: tráfico; regulación; control; políticas

\begin{abstract}
Despite the efforts of the various municipal administrations of the Metropolitan District of Quito, is very noticeable that the traffic of the city becomes more chaotic. Public policies have been implemented that have been taken as examples of other countries, without considering the real needs of the city, as is the case of "Pico y Placa", used in the Federal District of Mexico, and Bogota, and instead of solving the problem, have enabled the families of economic and high estratos, acquire one or more vehicles, in the same way, the resources invested by the municipalities for the control and regulation of traffic are becoming higher.
\end{abstract}

Key words: traffic; regulation; control; political 


\section{Introducción}

El transporte particular, es un tema que tiene importancia social tanto por el congestionamiento de la ciudad, como por las grandes cantidades de contaminación ambiental. Por otro lado, el mejoramiento, y ampliación de las vías, no constituye una única alternativa de solución, ya que es posible que aumente repentinamente el volumen de vehículos causando una congestión inesperada, contraria a todos los pronósticos de tráfico.

Se debe tratar entonces al problema desde el punto de vista de motivación comunitaria de uso del transporte público, y a la restricción de la demanda de transporte privado con el fin de solventar los efectos relacionados con el sistema de transporte.

Bajo la perspectiva de la Ciudad de Quito, se ha tratado de solucionar estos problemas planteados, solamente con experiencias de otros países, con escaso fundamento técnico, mucha de las veces por la escasez de recursos, o simplemente por la mala gestión en cuanto a Ingeniería de Tránsito. Es necesario entonces analizar los costos de funcionamiento, tiempos estimados de viaje, magnitudes, contaminación, entre otros, para llegar a formular una estrategia que permita regularizar el tránsito vehicular de la cuidad.

La ciudad de Quito cuenta con 2.576.287 habitantes según el último censo (Cifras, 2010) constituyéndose como la segunda ciudad más poblada del país, luego de Guayaquil. Por lo que la congestión vehicular ha hecho que en horas de alta demanda u horas pico, se vuelva prácticamente intransitable por la alta cantidad de vehículos particulares, taxis, buses colectivos, y buses de servicio escolar. Por otro lado, no se trata únicamente de congestión en vías, sino de contaminación ambiental y contaminación acústica.

Los problemas de congestión y contaminación en Quito, no es un tema reciente; la mala calidad de las vías, y la escasa conciencia de manejo de los ciudadanos, ha venido acarreando este problema desde tiempo atrás. Las distintas administraciones municipales han tratado de buscar soluciones a la saturación de las vías, implementando medidas que buscan incrementar la capacidad de éstas con el fin de lograr una mejor situación de la movilidad en la ciudad: ampliación de carriles, nuevas vías, intercambiadores viales, etc. Es por ello que se ha implementado desde el año 2010 el sistema de "Pico y Placa" que tiene como finalidad la restricción de la circulación de automotores particulares. La medida tiene como objetivo el desestimular a la ciudadanía el uso de transporte particular en los días que le corresponda la medida, y fomentar el uso de transporte público, con ello mejorar los tiempos de viaje por una menor cantidad de vehículos que circulen a esas horas de concentración vehicular.

De acuerdo al Plan Maestro de Movilidad, en el 2008, 60\% de las vías de la ciudad tenían una relación de volumen de tráfico a capacidad de la vía comprendida entre 0 y 0.8 , permitiendo una velocidad promedio de desplazamiento de más de $50 \mathrm{~km} / \mathrm{h}$. Mientras tanto, $25 \%$ de las vías tenía una relación volumen/capacidad de más de 1, es decir que estaban saturadas con una velocidad promedio de desplazamiento de $0-10 \mathrm{~km} / \mathrm{h}$. Para el 2015, dadas las actuales condiciones de crecimiento del parque automotor y de movilización, se prevé que $44 \%$ de las vías cuenten con una relación volumen/capacidad de 0-0.8 y que 38\% de las vías tenga una relación volumen/capacidad de más de 1. Este problema se agravaría aún más para el 2025, cuando se prevé que sólo $27 \%$ de las vías de la ciudad tengan una relación volumen/capacidad de 
0-0.8 y 55\% de las vías estén saturadas con una relación volumen/capacidad superior a 1. (Quito M. d., 2008)

\section{Método}

\section{Implementación del "Pico y Placa" en Quito}

Con esta situación mencionada, el Municipio Metropolitano de Quito el 3 de mayo del 2010 decidió implementar la medida de Pico y Placa, según la ordenanza 0305 de regulación de la circulación vehicular (Quito C. M., 2010), mediante los estudios técnicos que se detallarán más adelante.

Se determinó la distribución horaria promedio del flujo vehicular durante el día en la ciudad de Quito, dando como resultado que las horas pico de la ciudad son alrededor de las 8:00 y las 18:00 horas, por lo que la administración Municipal implementa la restricción a la circulación para dos números de placa por día (entendiéndose que se trata del último dígito de la placa), además que las sanciones por incumplir esta medida para este año estaban previstas en:

- $\quad$ por la primera vez de la infracción $\$ 113,33$;

- por la segunda, \$170;

- $\quad$ y por la tercera $\$ 340$,

Sin embargo, la actual administración el 31 de mayo del 2014, aprobó reducir esos valores al 50\% (Universo, 2014)

La medida funciona de la siguiente manera: para automóviles particulares la medida opera de lunes a viernes de 7:00 a 9:30 y de 16:00 a 19:30, cada día se restringe en las horas mencionadas a los vehículos que tuvieran placas terminadas en cualquiera de las dos cifras determinadas para cada día, con lo que el máximo de restricción sería del 20\% del parque automotor de vehículos particulares, tal como se observa en la tabla 1 las restricciones por número de placa.

Tabla 1. Números restringidos por el Pico y Placa
\begin{tabular}{llllll}
\hline Lunes & Martes & Miércoles & Jueves & Viernes \\
\hline $\mathbf{1}$ y 2 & 3 y 4 & 5 y 6 & 7 y 8 & 9 y 0 \\
\hline
\end{tabular}

Están exentos de la restricción a la circulación vehicular los siguientes:

a) Los vehículos oficiales del Presidente y Vicepresidente de la República, es decir aquellos que conforman la caravana de desplazamiento de dichas autoridades;

b) Los vehículos oficiales del cuerpo diplomático y consular que tengan placas de color azul con letras CD, CC, AT Y01;

c) Los vehículos de transporte de personas con discapacidad que cuenten con el salvoconducto que se detalla en este Reglamento;

d) Los vehículos conducidos por personas de la tercera edad, excepción que será validada en los operativos de control en la vía pública, a través de la presentación de la licencia de conducción y de la cédula de ciudadanía; 
e) Los vehículos que cumplen tareas de asistencia social y emergencias pertenecientes a instituciones del Estado, al igual que ambulancias privadas, debidamente identificadas;

f) Los vehículos de transporte colectivo de pasajeros en cualquiera de sus modalidades; es decir, público, escolar, institucional y turístico, debidamente autorizados por el Municipio, y también los que realizan recorridos por cuenta propia, en cuyo caso deberán registrarse en el Municipio;

g) Los vehículos que prestan el servicio de transporte de carga liviana en las parroquias rurales del Distrito, debidamente autorizados por el Municipio, que temporalmente ingresen al área urbana consolidada del Distrito Metropolitano de Quito; y,

h) Los taxis legalmente autorizados por el Municipio. (Quito C. M., 2010)

\section{Impacto de la medida implementada}

En esta sección se estudia el impacto de la medida de pico y placa en la ciudad de Quito, analizando los flujos vehiculares, con y sin pico y placa, la efectividad de la medida en el tiempo y la relación con el impacto ambiental que ha tenido la implementación de la medida.

\section{Corredores Viales}

La medida implementada está comprendida dentro de los límites viales de referencia descritos a continuación e ilustrados en la figura 1.

$\checkmark$ Por el occidente: Av. Mariscal Sucre, entre Av. Morán Valverde y Av. Manuel Córdova Galarza (tramo vial con restricción); Av. Mariscal Sucre entre Av. Manuel Córdova Galarza y Av. Diego Vásquez de Cepeda (tramo sin restricción).

$\checkmark$ Por el norte: Av. Diego Vásquez de Cepeda, entre Av. Galo Plaza y Av. Manuel Córdova Galarza (tramo vial sin restricción); y, Panamericana Norte, entre la Av. Simón Bolívar y Av. Galo Plaza (tramo vial sin restricción).

$\checkmark$ Por el oriente: Av. Simón Bolívar (tramo vial sin restricción).

$\checkmark$ Por el sur: Av. Morán Valverde, entre Av. Simón Bolívar y Av. Mariscal Sucre (vía sin restricción).

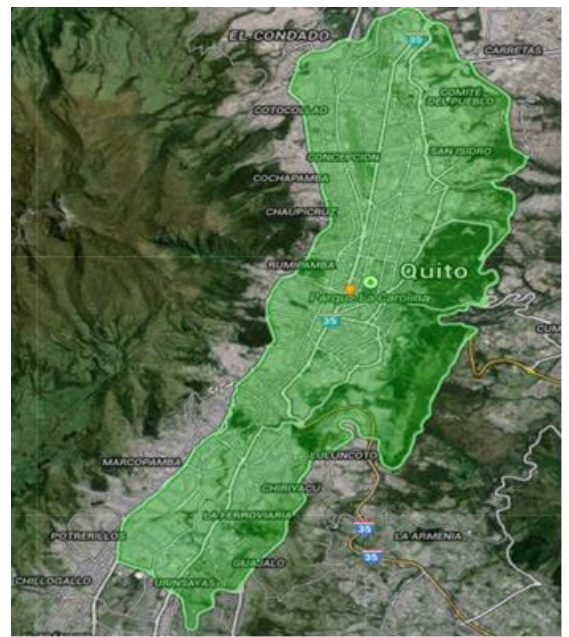

Figura 1. Delimitación de la zona Pico y Placa en el DMQ 
Las avenidas Morán Valverde, Simón Bolívar, Panamericana Norte y Diego Vásquez de Cepeda (en los tramos antes indicados) se constituyen al mismo tiempo en límites como en ejes viales sin restricción de circulación, de manera que permiten el cruce perimetral de la ciudad para los viajeros de largas distancias, tanto hacia el norte y sur, como al noroccidente del país. (Quito C. M., 2010)

\subsubsection{Estudio Técnico}

El 10 de junio del 2010, el Municipio de Quito mediante el portal de compras públicas se contrató a la Facultad de Arquitectura de la Universidad Central del Ecuador para que realice varios estudios de tráfico, necesarios para determinar algunos índices de movilidad urbana, y que permitan evaluar los resultados de la medida de restricción Vehicular, denominada Pico y Placa.

El estudio consta de tres fases, del 1 al 30 de junio la Fase 1 que corresponde al estudio del tráfico antes de la aplicación de la medida. Del 1 de julio al 31 de agosto la fase II que corresponde a los 3 meses de aplicarse la medida, y del 1 de octubre al 30 de noviembre la Fase III que corresponde a los 6 meses de aplicarse la medida. Todas estas en el año 2010.

\section{Parque Automotor de Quito}

Según la Secretaría de Movilidad la información con mayor proximidad para expresar el patrón de crecimiento del parque vehicular de Quito es la Revisión Técnica Vehicular que lleva adelante la municipalidad.

Al 2013, el parque automotor en el DMQ estuvo compuesto por cerca de 550.000 vehículos. El crecimiento del parque vehicular es de 9,2\% anual, que corresponde a un incremento anual cercano a los 50.000 vehículos; cuatro veces superior al crecimiento poblacional. Esto significa que al 2011 la tasa de motorización en el DMQ alcanzaba un promedio de 15 autos por cada 100 habitantes y 51 vehículos por cada 100 hogares (Ciudad, 2014)

De acuerdo al Plan Maestro de Movilidad 2008- 2025, la red vial de Quito presenta actualmente una saturación del $30 \%$ debido a altos volúmenes de tráfico que superan la capacidad vial, especialmente en horas pico. El parque automotor crece en un promedio de 40,000 nuevas unidades por año. Así, la proporción de autos por mil habitantes aumentó de 145 en 2002 a 180 en 2007 y, si se mantiene este ritmo de crecimiento, se llegará a 590 vehículos por mil habitantes en 2025. Adicionalmente, el Plan Maestro también señala que la población de la ciudad se encuentra en crecimiento (Mosquera, 2010)

\section{Índices de Tráfico}

\section{Resultados}

- Antes de la medida 
El volumen total diario de tráfico que se tomaron en base a los puntos de control establecidos por el estudio muestra que hay una reducción de un 7,5\% en vehículos particulares, y de $15 \%$ en motocicletas, sin embargo, se observa un aumento del $10 \%$ en circulación de taxis.

- Después de la medida

Durante los horarios de vigencia del Pico y Placa, hay una reducción del $12 \%$ en vehículos particulares, $25 \%$ para camiones y transporte pesado y $17 \%$ para motocicletas, Además de un aumento de $12 \%$ de taxis. Estos datos demuestran que existe un $8 \%$ de reducción de tráfico pico en la mañana, y un $12 \%$ en el tráfico pico de la tarde. Se debe tomar en cuenta que antes de la hora de la medida (07:00) se aprecia un significativo aumento de 8\% para vehículos particulares, y hasta un $29 \%$ para motocicletas.

\section{Velocidades de Circulación}

Los datos que a continuación se presentan, son tomados de los estudios realizados por la Universidad Central. (Ecuador, 2010)

En la Autopista Rumiñahui, la velocidad promedio de circulación en el horario de aplicación de la medida es en promedio $50 \mathrm{Km} / \mathrm{h}$.

$\checkmark$ En la Avenida Mariscal Sucre, la velocidad promedio es de $40 \mathrm{Km} / \mathrm{h}$.

$\checkmark$ En la Avenida Eloy Alfaro, se observó una velocidad promedio de $25 \mathrm{Km} / \mathrm{h}$.

$\checkmark$ En la Avenida de los Shirys, un promedio de $33 \mathrm{Km} / \mathrm{h}$.

$\checkmark$ La Avenida Maldonado, un promedio de $30 \mathrm{Km} / \mathrm{h}$.

$\checkmark$ La calle García Moreno, una de las más caóticas, tiene una velocidad promedio de $15 \mathrm{Km} / \mathrm{h}$.

En las Intersecciones entre las Avenidas El Inca y 6 de diciembre, se mantiene las longitudes de filas de vehículos, sin un decrecimiento significativo.

\section{Niveles de Contaminación}

La medida muestra efectividad durante las horas de aplicación en cuanto a la reducción de concentraciones de $\mathrm{CO}$ en el aire: $0,16 \mathrm{mg} / \mathrm{m} 3$ por la mañana y entre 0,05 y $0,09 \mathrm{mg} / \mathrm{m} 3$ por la tarde y lo mismo para material particulado que disminuye gracias al aumento de la velocidad de los vehículos, y no por las concentraciones medidas en el escape de los vehículos pesados.

\section{Conclusiones}

Debido al veloz incremento del parque Automotor, se puede afirmar que la medida Pico y Placa solucionó los problemas en los 3 primeros años de aplicación, pero luego de ello, esta medida está siendo obsoleta, por lo que se deben buscar nuevas alternativas de solución al tráfico vehicular, ofreciendo soluciones alternativas, como es el fomentar el uso de un transporte público accesible, eficiente y seguro, además de proponer soluciones no motorizadas como es el uso de la bicicleta. 
Por otro lado, el pensar en ampliar la medida a lo largo del día, es viable, siempre y cuando se oferte a la ciudadanía un transporte público eficiente, que aliente a dejar el vehículo particular en casa. Pese a la implementación de la medida Pico y Placa, las velocidades de circulación en calles y avenidas es relativamente bajo, disminuyendo también por la falta de conciencia de conductores que parquean los vehículos en la vía. En lo que se refiere a los niveles de contaminación por material particulado, no disminuye en cantidad, sino en medición debido al incremento de la velocidad de circulación de buses. Los estudios realizados por la Universidad Central muestran que la medida Pico y Placa tiene poco impacto, principalmente por el hecho de haber realizado los estudios técnicos en un tiempo de 6 meses.

El fenómeno descrito muestra la tendencia del Pico y Placa a distribuir la congestión a lo largo del día, haciendo que los tiempos de viaje y las velocidades de recorrido en las horas pico mejoren, pero aumenten en las horas normales, de hecho, es común el comentario de que en las tradicionales horas pico hay más fluidez vehicular que en las demás horas del día, por lo que ya no son horas pico

\section{Bibliografía}

Cifras, E. e. (2010). INEC. (Gobierno Nacional de la República del Ecuador) Obtenido de http://www.ecuadorencifras.gob.ec/censo-de-poblacion-y-vivienda/

Ciudad, I. d. (01 de 13 de 2014). www.institutodelaciudad.com.ec. Obtenido de Boletín estadístico No. 21: http://institutodelaciudad.com.ec/attachments/article/149/boletin21.pdf

Ecuador, U. C. (20 de 12 de 2010). Estudio para la determinación de ínices de movilidad Urbana para evaluar los impáctos de la medida de restricción vehicular Pico \& Placa en la ciudad de Quito. Obtenido de http://www.noticiasquito.gob.ec/modules/umFileManager/pndata/test/informe_pp_iii_3500. pdf

Mosquera, R. (01 de 05 de 2010). Racionalización Del Uso Del Automóvil En Quito. Obtenido de http://www.udla.edu.ec/wp-content/uploads/2013/12/1432011130Racionalizacion-del-usodel-automovil-en-Quito-final.pdf

Quito, C. M. (28 de 04 de 2010). www.policiametropolitanaquito.gob.ec. Obtenido de http://policiametropolitanaquito.gob.ec/lotaip/ordenanzas/ORDM-305\%20$\% 20$ circulacion \%20vehicular\%20-\%20pico\%20y\%20placa.pdf

Quito, M. d. (2008). Plan Maestro de Movilidad 2008-2025. Obtenido de http://urbegestion.com/images/Documentos/Muni_Quito_2009_Plan_Maestro_Movilidad_2 009-2025_Presentacion.pdf

Universo, E. (31 de 05 de 2014). Se baja 50\% en multas de pico y placa y la zona azul en Quito. El Universo. 